\title{
Pimavanserin, a Serotonin $2 A$ Receptor Inverse Agonist, for the Treatment of Parkinson's Disease Psychosis
}

\author{
Herbert Y Meltzer*,', Roger Mills², Stephen Revell ${ }^{2}$, Hilde Williams², Ann Johnson ${ }^{2}$, Daun Bahr ${ }^{2}$ \\ and Joseph H Friedman ${ }^{3}$ \\ 'Department of Psychiatry, Vanderbilt University School of Medicine, Psychiatric Hospital at Vanderbilt, Nashville TN, USA; ${ }^{2}$ ACADIA \\ Pharmaceuticals, San Diego, CA, USA; ${ }^{3}$ Department of Clinical Neurosciences, The Warren Alpert School of Medicine of Brown University, \\ Parkinson's Disease and Movement Disorders Center, NeuroHealth, Warwick, RI, USA
}

\begin{abstract}
Psychotic symptoms occur in up to $40 \%$ of patients with Parkinson's disease (PD). Clozapine and quetiapine, two atypical antipsychotic drugs, at doses markedly lower than those effective in schizophrenia, which, nevertheless, still cause sedation, hypotension, and other side effects, are widely used to treat psychotic symptoms in patients with PD psychosis (PDP), although quetiapine has never been shown to be effective in a placebo-controlled study. The demonstrated efficacy of clozapine in PDP has been attributed to serotonin (5-HT $2 \mathrm{~A}$ ) receptor blockade. We postulated that pimavanserin (ACP-103), a highly selective $5-\mathrm{HT}_{2 \mathrm{~A}}$ inverse agonist, would attenuate psychosis in patients with PDP, but avoid motoric worsening and non-motoric side effects. In this double-blind, randomized multicenter 28-day study, the tolerability and efficacy of pimavanserin was compared with placebo in 60 patients with L-DOPA or dopamine (DA) agonist-induced PDP. Motor function was evaluated using the Unified Parkinson's Disease Rating Scale (UPDRS) Parts II and III. Antipsychotic efficacy was evaluated using multiple measures from the Scale for the Assessment of Positive Symptoms (SAPS) and a UPDRS Part I psychosisrelevant item. Pimavanserin did not differentiate from placebo with regard to motor impairment, sedation, hypotension, or other side effects. The principal measures of efficacy of antipsychotic response to pimavanserin, the SAPS total domain score, only showed a trend. However, the pimavanserin-treated patients showed significantly greater improvement in some but not all measures of psychosis, including SAPS global measures of hallucinations and delusions, persecutory delusions, and the UPDRS measure of delusions and hallucinations. Pimavanserin showed significantly greater improvement in psychosis in patients with PDP at a dose which did not impair motor function, or cause sedation or hypotension Thus, pimavanserin may represent a novel treatment for PDP. Furthermore, these results support the hypothesis that attenuation of psychosis secondary to DA receptor stimulation in PDP may be achieved through selective $5-\mathrm{HT}_{2 \mathrm{~A}}$ receptor antagonism.
\end{abstract}

Neuropsychopharmacology (20I0) 35, 88I-892; doi:I0.1038/npp.2009. I76; published online II November 2009

Keywords: pimavanserin (ACP-103); Parkinson's disease; hallucinations; delusions; motor symptoms

\section{INTRODUCTION}

Psychotic symptoms, particularly visual hallucinations and paranoid delusions, occur in up to $40 \%$ of patients with Parkinson's disease (PD) who receive dopamine (DA) replacement therapy (Chou et al, 2007; Fenelon et al, 2000; Fernandez et al, 2003: Friedman, 1991, Goetz et al, 2001; Weintraub and Stern, 2005; Ravina et al, 2007; Diederich et al, 2009). Psychotic symptoms in PD psychosis (PDP) are a significant cause of distress to patients and their caregivers, and are associated with greater functional

*Correspondence: Dr HY Meltzer, Department of Psychiatry, Vanderbilt University School of Medicine, Psychiatric Hospital at Vanderbilt, I60I 23rd Ave South, Suite 306, Nashville, TN 37212, USA, Tel: + 6153277049 , Fax: + 6153277093

E-mail: Herbert.meltzer@vanderbilt.edu

Received 24 May 2009; revised 15 September 2009; accepted 16 September 2009 impairment, caregiver burden, nursing home placement, and increased mortality (Weintraub and Stern, 2005; Factor et al, 2003). As a result, antipsychotic drug treatment is often used to manage persistent and troublesome psychotic symptoms in PD patients (Chou et al, 2007; Fenelon et al, 2000; Fernandez et al, 2003). It has been suggested that psychotic symptoms in PD result from stimulation of limbic system DA $\mathrm{D}_{2}$ receptors (Romrell et al, 2003), as is believed to be the case in schizophrenia (Meltzer and Stahl, 1976). Typical antipsychotic drugs are intolerable as a treatment for PDP because, at the doses needed to block limbic $D_{2}$ receptors, they also block dorsal striatal $D_{2}$ receptors, reducing the ameliorative effects of L-DOPA or direct acting DA agonist treatment on motor symptoms (Chou et al, 2007; Romrell et al, 2003). Low doses of risperidone and olanzapine, two atypical antipsychotic drugs with diminished liability to cause EPS, also worsen motor symptoms in PDP, even at the minimal doses needed 
to treat psychotic symptoms (Molho and Factor, 1999; Weintraub and Stern, 2005).

Clozapine, an atypical antipsychotic drug, is the only antipsychotic that has shown efficacy and tolerability in placebo-controlled studies in patients with PDP (Parkinson Study Group, 1999). It has been hypothesized that clozapine, at doses which are effective in PDP (6.25$75 \mathrm{mg} /$ day), $3-10 \%$ of the doses usually used to treat schizophrenia, does not sufficiently block limbic DA $\mathrm{D}_{2}$ receptors to achieve an antipsychotic effect through $\mathrm{DA} \mathrm{D}_{2}$ receptor blockade, and that a more likely basis for its antipsychotic activity in PDP is serotonin $(5-\mathrm{HT})_{2 \mathrm{~A}}$ receptor blockade (Meltzer et al, 1995). This is consistent with abundant evidence that hallucinations can result from the stimulation of cortical $5-\mathrm{HT}_{2 \mathrm{~A}}$ receptors and can be blocked by $5-\mathrm{HT}_{2 \mathrm{~A}}$ inverse agonists or antagonists (Nichols, 2004), and that $5-\mathrm{HT}_{2 \mathrm{~A}}$ antagonism is integral to the antipsychotic properties of multireceptor acting atypical antipsychotic drugs, such as clozapine and quetiapine (Meltzer et al, 2003).

Despite its demonstrated effectiveness, the use of clozapine in PDP is limited because of sedation, hypotension, hypersalivation, and the risk of agranulocytosis (Chou et al, 2007; Fernandez et al, 2003; Weintraub and Stern, 2005). Quetiapine, another atypical antipsychotic drug, has been reported in a number of open-label studies to reduce psychosis in PD without worsening motor symptoms, but sedative and hypotensive effects also limit its tolerability (Fernandez et al, 1999, 2003; Reddy et al, 2002; Mancini et al, 2004; Morgante et al, 2004; Merims et al, 2006). Furthermore, in three placebo-controlled, double-blind, randomized trials, quetiapine failed to show superiority to placebo on measures of psychosis in PDP (Ondo et al, 2005; Rabey et al, 2006; Shotbolt et al, 2009). Nevertheless, it remains widely used as the initial drug treatment for PDP, at doses from 25 to $300 \mathrm{mg} / \mathrm{day}$, and is the drug of choice for PDP according to the American Academy of Neurology Practice Parameters Task Force on the treatment of PD (Miyasaki et al, 2006); clozapine is recommended only after quetiapine fails to manage psychotic symptoms (Chou et al, 2007; Reddy et al, 2002; Mancini et al, 2004). Recent reports of increased mortality in elderly demented patients treated with various antipsychotic drugs (Wang et al, 2005; Ballard et al, 2009) raises additional concerns about the safety of these drugs in patients with PDP and supports the need for the development of safer treatments.

The sedative and hypotensive effects of clozapine and quetiapine likely result from histamine $\mathrm{H}_{1}$ receptor and alpha $_{1}$ adrenoceptor blockade (Arana, 2000). Pimavanserin is a novel selective $5-\mathrm{HT}_{2 \mathrm{~A}}$ receptor inverse agonist, which has shown antipsychotic activity in animal models, including blockade of amphetamine- or the $N$-methyl-D-aspartate receptor noncompetitive antagonist $5 \mathrm{H}$-dibenzo[a,d]cyclohepten-5,10-imine (dizocilpine maleate; MK-801)-induced locomotor activity, conditioned avoidance response, head twitch, and the deficit in prepulse inhibition produced by the $5-\mathrm{HT}_{2 \mathrm{~A}}$ agonist (+/-)-2,5-dimethoxy-4-iodoamphetamine hydrochloride (DOI) in rats (Vanover et al, 2006). Pimavanserin has approximately 40-fold lower affinity for the $5-\mathrm{HT}_{2 \mathrm{C}}$ than the $5-\mathrm{HT}_{2 \mathrm{~A}}$ receptor, and there is no measurable activity at other G-protein-coupled receptors (Vanover et al, 2006). On the basis of its specificity for the
$5-\mathrm{HT}_{2 \mathrm{~A}}$ receptor, it was, therefore, of interest to test pimavanserin as a treatment for PDP and to evaluate its safety and tolerability profile in this population. The results reported here indicate that pimavanserin has a benign profile on motor function and other safety parameters, and was more effective than placebo in attenuating hallucinations and delusions in patients with PDP.

\section{PATIENTS AND METHODS}

\section{Study Patients}

Patients who met criteria for PDP, consistent with the criteria developed by Ravina et al (2007), including the presence of visual and/or auditory hallucinations, and/or delusions, of moderate-to-severe frequency, and severity, for at least 4 weeks, were screened for eligibility. The minimum requirement for entry was a psychosis severity score of $\geqslant 4$, as assessed by the hallucinations and delusions sections of the Neuropsychiatric Inventory (NPI) (Cummings et al, 1994). Patients were required to be on stable antiparkinsonian drugs for at least 1 week before study entry and through the 4 -week treatment period. Fifteen patients in the pimavanserin arm and 15 in the placebo arm received L-DOPA only; 11 in the pimavanserin arm and 19 in the placebo arm received L-DOPA plus a direct acting DA agonist. No other antipsychotic medications were permitted. Exclusion criteria included pregnancy, presence of any other medical condition contributing to psychosis, significant pre-morbid psychiatric condition other than a history of major depression before the diagnosis of PD, dementia, or myocardial infarction within the past 3 months. Medications required for stable medical conditions were permitted and did not change during the course of the study. Written informed consent was obtained from all patients and/or their caretakers before performing study procedures.

\section{Study Design}

This Institutional Review Board approved phase 2 multicenter study was a randomized, placebo-controlled, doubleblind trial of 4 weeks duration, with a 4 -week follow-up period. Patients were randomly assigned to receive pimavanserin or placebo at a ratio of $1: 1$ after completion of the screening and baseline evaluations, according to a centerspecific, computer-generated randomization code. Thirteen sites enrolled at least two patients; two sites enrolled only one. Patients received study drug daily for 4 weeks, starting at $20 \mathrm{mg}$ on study day 1 , with possible increases to 40 or $60 \mathrm{mg}$ daily doses on study days 8 and 15, respectively, depending on individual clinical response.

Patients were evaluated at screening/baseline (up to 14 days before study day 1) and on study days $1,8,15,28$, and 57 (safety data only). Physical exams, vital signs, and laboratory tests were completed during each clinic visit. Adverse events were recorded and categorized for severity and relationship to study treatment from day 1 to 57 . Study day 57 or early termination was included as a safety followup visit. Later, patients were given the option of extended open-label treatment with pimavanserin under a separate protocol. 


\section{Safety Measures}

The modified Hoehn and Yahr (UPDRS Part V) staging of PD was completed at screening. The primary measures used to assess motor symptoms were the Unified Parkinson's Disease Rating Scale (UPDRS) Parts II (Activities in Daily Living) and III (Motor Examination) (Goetz et al, 2007). Additional safety assessments included an adverse events check list, vital signs, laboratory tests (hematology, clinical chemistry, and urinalysis), physical examinations, and electrocardiograms (ECG).

\section{Efficacy Measures}

Psychosis was assessed by the Scale for the Assessment of Positive Symptoms (SAPS; Andreasen, 1984), the Parkinson's Psychosis Rating Scale (PPRS; Friedberg et al, 1998), and the Clinical Global Impression-Severity (CGI-S; Guy, 1976). In addition, the UPDRS Parts I, IV, and VI were used to evaluate the effects of treatment on mentation, behavior, and mood; complications of therapy; and activities of daily living, respectively (Goetz et al, 2004), Daytime sleepiness was measured by the Epworth Sleepiness Scale (ESS Johns, 1991). Rater training was provided before study initiation.

A specified analysis of the SAPS domain scores for hallucinations and delusions and their combination was conducted. The domain score is the sum of the individual ratings for each type of symptom within the domain, and includes the global rating for that domain (Andreasen, 1984). The SAPS total domain score was chosen as principal outcome measure for efficacy because it was the one used in the study of the efficacy of clozapine in PDP (Parkinson's Study Group, 1999). Other planned analyses of the SAPS data included comparisons of the change from baseline to day 28 in each individual item and the global ratings of hallucinations and delusions. The global ratings of hallucinations and delusions were also combined to generate a total global score.

Other planned analyses for the secondary efficacy measures: PPRS, CGI-S, Epworth Daytime Sleepiness Scale, and UPDRS Parts I, IV, and VI were also conducted. An exploratory analysis of the UPDRS Part I items (intellectual impairment, thought disorder (a measure of psychosis), depression, and motivation) was also completed.

All of the efficacy measures described above were treated as continuous variables and were analyzed using analysis of covariance (ANCOVA). The change from baseline to day 28 scores was examined by ANCOVA with treatment and center as main effects and the baseline score of the respective measure included as the covariate in the model. Higher scores indicate greater severity of symptoms with the exception of UPDRS Part VI, in which higher scores indicate improved functioning in activities of daily living. Negative difference scores (day 28 to baseline) indicate improvement.

\section{Statistical Analysis}

This study was $95 \%$ powered to detect a relative difference of five points in the UPDRS Part II and III scores between the treatment groups in the absolute change from baseline to the last observation completed. This analysis was based on prior clinical trials that have documented the motoric effects of clozapine (Parkinson's Study Group, 1999; The French Clozapine Parkinson's Study Group, 2003) and olanzapine (Goetz et al, 2000) in PDP. On the basis of this assumption, 54 patients (27 per arm) were required to complete the study. To adjust for a $10 \%$ dropout rate, $\sim 60$ patients were to be enrolled and randomized $(\sim 30$ per arm). The primary safety analysis was the change from baseline to day 28 in the UPDRS Part II and III total score using last observation carried forward (LOCF) with an analysis of covariance (ANCOVA) model, with treatment and center as main effects, performed to compare the treatment groups. The per-protocol (PP) population was used for this analysis.

The ITT population included 59 of the 60 enrolled patients (28 receiving pimavanserin and 31 receiving placebo) who had met entry criteria, had taken at least one dose of trial medication, and had at least one postbaseline efficacy outcome measure. One patient (patient 11-003) was dropped from the ITT population as he did not meet the entry criteria (lung disorder), which was not determined until after dosing. In addition to the primary ITT-LOCF analysis, a similar analysis was carried out at study day 28 using observed cases (OCs) in the PP population, which included 52 patients ( 24 patients receiving pimavanserin and 28 patients receiving placebo) from the ITT population who had met entry criteria, and recorded no major deviations from protocol, including completion of the study day 28 visit with at least $70 \%$ overall treatment compliance. Patients in the PP population were classified according to the treatment they received, two patients accidentally received treatment not according to the randomization list. The PP-OC analysis was considered the most appropriate for analysis of the primary safety measure, UPDRS, which is a non-inferiority assessment (Lewis, 1999). The PP-OC analysis was used on all secondary end points because these were considered to be exploratory in nature. Therefore, no adjustments for multiple comparisons were made in the reporting of $p$-values. Results of the ITT-LOCF analysis of secondary end points were generally similar to those of the PP-OC analysis. Standardized effect size, defined as the difference in adjusted treatment means divided by the pooled standard deviation, was calculated concurrently with $p$-values.

All available safety data from the 60 enrolled patients receiving at least one dose of study medication (29 receiving pimavanserin and 31 receiving placebo) were included in the safety analysis. Patients in the safety population were classified according to the treatment they received.

Descriptive statistics ( $N$, mean, $\mathrm{SD}$, coefficient of variance (CV\%), median, minimum, and maximum) of values and change from baseline values were used to summarize laboratory, vital sign, and ECG data over time. Homogeneity of baseline characteristics was assessed by $t$-test for continuous variables, Fisher's exact test for categorical variables.

\section{RESULTS}

\section{Patients}

As shown in Figure 1, 60 patients were enrolled; of them, 28 of 29 patients in the pimavanserin group and 28 of 31 


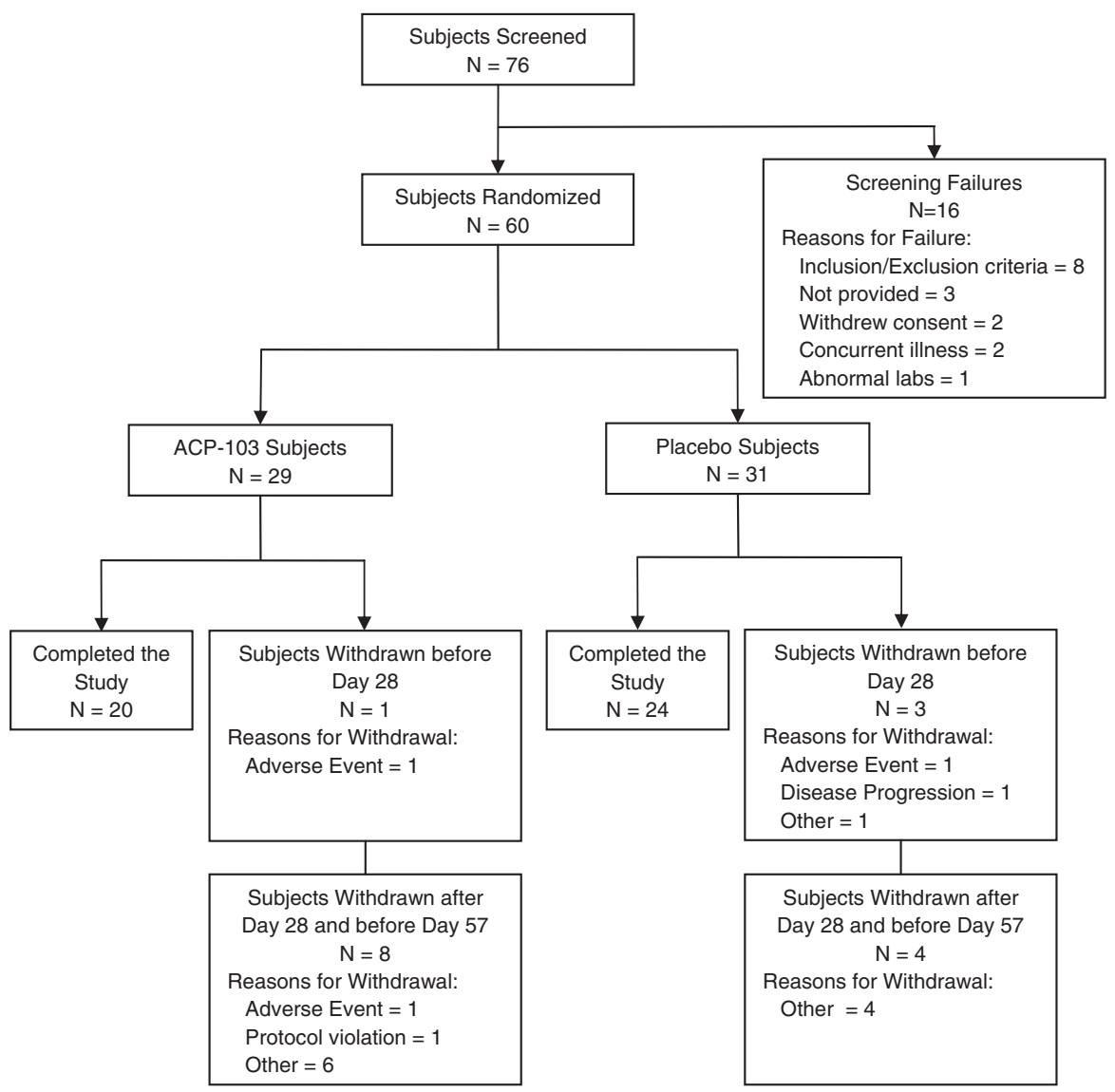

Figure I Flowchart of the study population.

patients in the placebo group completed 28-day study. The mean (SD) final dose of pimavanserin and placebo equivalent administered was 44.8 (16) and 52.9 (12) $\mathrm{mg}$, respectively. Of the 28 pimavanserin subjects reaching day 28, 20 remained in the study for safety follow-up through day 57, one was terminated because of an adverse event, one for a protocol violation, and four for other reasons. Of the 28 placebo subjects reaching day 28,24 completed the safety follow-up visit at day 57. All remaining four placebo subjects dropped out because of 'other reasons.' Ratings of psychotic symptoms were not conducted during this period.

Demographic and baseline characteristics are summarized in Table 1. Age, ethnicity, and race were similar in both study groups. There were fewer females $(N=3,10.3 \%)$ and more males $(N=26,89.7 \%)$ in the pimavanserin group than in the placebo group $(11,35.5 \%$, and $20,64.5 \%$, respectively, $p=0.03)$. At entry, the mean NPI total scores of delusions and hallucinations and the Modified Hoehn and Yahr Staging scores were not statistically different $(p=0.35$ and 1.0 , respectively).

\section{Effects on Motor Function}

At day 28, there was a small nonsignificant improvement in both treatment groups in the combined score of UPDRS, Parts II (Activities of Daily Living) and III (Motor Function): adjusted mean changes of -3.05 for pimavan- serin and -3.86 for placebo (Table 2). No statistically significant differences were observed in treatment effect $(p=0.74,95 \%$ CI: $-4.18,5.80)$. Similar results were observed for the UPDRS Parts II and III scores analyzed as separate scores (Table 2).

\section{Effects on Psychosis}

Table 3 provides the baseline and day 28 scores for the SAPS hallucination and delusion individual item scores, global, and total domain measures in the PP (OCs) population. The results of the statistical analysis for these measures are also presented in Table 3.

As expected, visual, followed by auditory hallucinations, was the most severe form of hallucinations at baseline (Table 3). There was a statistically significant improvement in the global rating of hallucinations in the pimavanserintreated patients $(p=0.02$, effect size $=0.58)$. Although the hallucinations domain score for the pimavanserin-treated patients showed improvement, it was not significant $(p=0.16)$.

At baseline, persecutory delusions were the most frequent and severe form of delusions, followed by delusions of jealousy (Table 3 ). There was significantly greater improvement in the pimavanserin-treated patients in the following SAPS delusion domain measures: persecutory delusions $(p=0.009$, effect size $=0.41)$, ideas and delusions of 
Table I Demographic and Baseline Characteristics - Safety Population

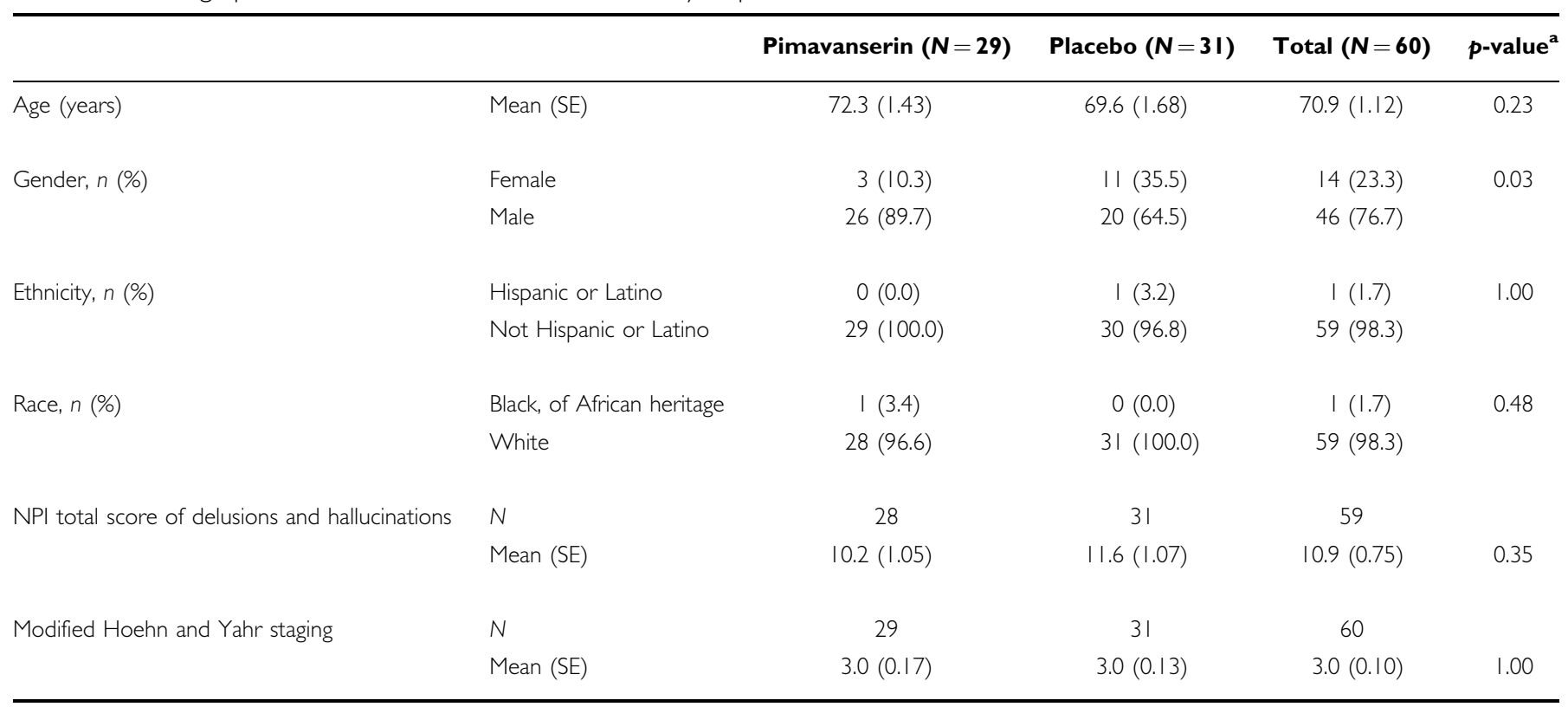

${ }^{a} p$-value is based on $t$-test for continuous variables and Fisher's exact test for categorical variables.

$p$-values are based on non-missing data.

Percentages are based on the number of patients in the Safety population.

Table 2 UPDRS Scores: Change from Baseline to Day 28 and Estimated Treatment Effects for the PP-OC Patient Population

\begin{tabular}{|c|c|c|c|c|c|c|c|}
\hline \multirow{2}{*}{ Outcome } & \multicolumn{2}{|c|}{ Pimavanserin } & \multicolumn{2}{|c|}{ Placebo } & \multicolumn{3}{|c|}{ Estimated treatment effect } \\
\hline & LSM* & $95 \% \mathrm{Cl}$ & LSM* & $95 \% \mathrm{Cl}$ & Difference in LSM & $95 \% \mathrm{Cl}$ & $p$-value \\
\hline Part III & -0.99 & $-3.72,1.74$ & -2.64 & $-5.29,0.01$ & 1.65 & $-2.25,5.55$ & 0.40 \\
\hline Parts II and III & -3.05 & $-6.56,0.46$ & -3.86 & $-7.20,-0.53$ & -0.81 & $-4.18,5.80$ & 0.74 \\
\hline
\end{tabular}

Abbreviations: Cl, confidence interval; LSM, least square mean; OC, observed case; PP, per protocol; UPDRS, Unified Parkinson's Disease Rating Scale.

Negative numbers represent improvement.

reference ( $p=0.05$, effect size $=0.36)$, and global ratings of delusions $(p=0.03$, effect size $=0.53)$. There was a trend for the SAPS delusion domain score to show improvement in the pimavanserin arm $(p=0.06$, effect size $=0.56)$. The total global rating, a more specific measure of functional improvement in delusions (Andreasen, 1984), showed significantly greater improvement with pimavanserin treatment ( $p=0.02$, effect size $=0.66$ ). There was also a trend for the pimavanserin-treated patients to show greater improvement in the SAPS total domain score $(p=0.09$, effect size $=0.52$ ). This reflected a $40 \%$ improvement in this measure in the pimavanserin-treated patients compared with a $11 \%$ improvement in the placebo-treated group.

The UPDRS Part I total score, similar to the SAPS global scores, showed significantly greater improvement in the pimavanserin-treated patients at day 28 (Table $4, p=0.05$, effect size $=0.43$ ). In particular, the thought disorder item showed significantly greater improvement in the pimavanserin-treated patients $(p=0.05$, effect size $=0.40)$. The anchor point description of the thought disorder rating item specifically denotes delusions and hallucinations as the key behaviors to be assessed in rating this item. In support of this, the UPDRS thought disorder rating was significantly correlated with both the SAPS global hallucinations and global delusions ratings at baseline $(r=0.38$ and 0.38 , $p=0.005$ and 0.006 , respectively $)$ and at day $28(r=0.68$ and $0.44, p=<0.001$ and 0.001 , respectively), as were the change from baseline to day 28 ( $r=0.48$ and $0.43, p=<0.001$ and 0.002 , respectively). The other UPDRS Part 1 items, which do not measure psychotic symptoms, also favored pimavanserin, but were not significantly different from placebo.

Other measures of psychosis, PPRS and CGI-S, showed improvements in pimavanserin-treated patients compared with placebo (Table 4); however, these comparisons were not statistically significant. After completion of the study, it was determined that there were inconsistencies in the way the CGI-S scale was administered at different sites, with some assessing changes in psychosis and other changes in overall condition. Improvements in measures of daytime sleepiness (Epworth Sleepiness Scale), complications with PD therapy (UPDRS Part IV), and activities of daily living (UPDRS Part VI) were also observed in pimavanserintreated patients compared with placebo (Table 4), although none of the comparisons achieved statistical significance. 
Table 3 ACP-103-006: Mean (SD) Baseline and Day 28 SAPS Scores; Change from Baseline (Screening Visit) to Day 28: Analysis of Covariance (ANCOVA); Per-Protocol Population (Observed Case)

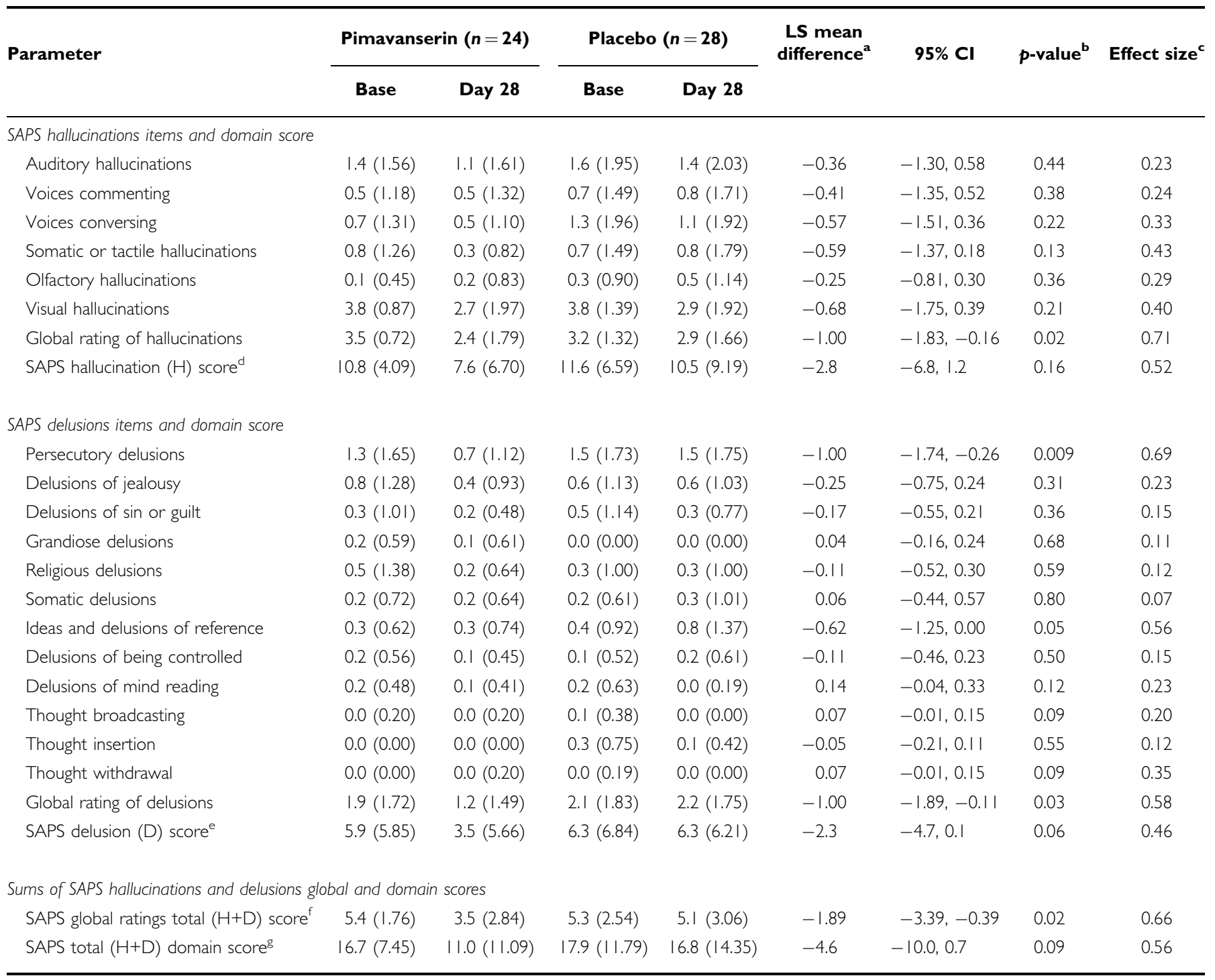

Abbreviation: SAPS, Scale for the Assessment of Positive Symptoms.

a Difference in LS means (pimavanserin-placebo).

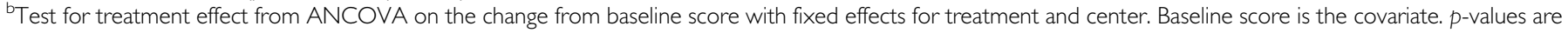
not adjusted for multiple comparisons.

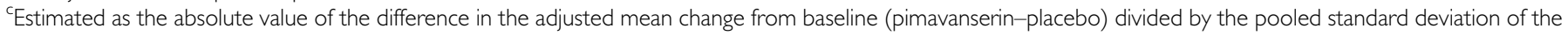
mean change from baseline score.

'Sum of all seven items for hallucinations.

esum of all 13 delusion items.

f Sum of global ratings for hallucinations and delusions.

'Sum of Domain scores for hallucinations and delusions; primary measure of efficacy.

*Negative numbers indicate greater improvement in the pimavanserin-treated group.

\section{Safety}

Table 5 provides a summary of adverse events. Overall, there was no significant difference in the incidence of adverse events in the placebo- and pimavanserin-treated patients. In total, 133 treatment-emergent adverse events were reported in $21(72.4 \%)$ patients receiving pimavanserin and $24(77.4 \%)$ patients receiving placebo. Fifty-one of the treatment-emergent adverse events were considered to be treatment-related.
In the pimavanserin arm, the most commonly occurring adverse events (present in $\geqslant 10 \%$ of patients) were somnolence, edema, and increase in blood urea nitrogen, each of which were reported in three $(10.3 \%)$ patients. Somnolence resolved in one case despite continuing pimavanserin. In another, it occurred 10 days after the last dose of pimavanserin. One patient treated with pimavanserin discontinued the study because of worsening of hallucinations.

In the placebo arm, the most commonly occurring adverse events (present in $\geqslant 10 \%$ of patients) were 
Table 4 ACP-103-006: Change from Baseline (Screening Visit) to Day 28: Analysis of Covariance (ANCOVA); Per-Protocol Population (Observed Case)

\begin{tabular}{|c|c|c|c|c|}
\hline Parameter & LS mean difference ${ }^{a, b}$ & $95 \% \mathrm{Cl}$ & $p$-value ${ }^{c}$ & Effect size ${ }^{d}$ \\
\hline UPDRS Part 1 & -1.45 & $-2.89,-0.01$ & 0.05 & 0.43 \\
\hline Intellectual impairment & -0.30 & $-0.81,0.22$ & 0.25 & 0.34 \\
\hline Thought disorder & -0.57 & $-1.15,0.01$ & 0.05 & 0.58 \\
\hline Depression & -0.31 & $-0.77,0.15$ & 0.18 & 0.41 \\
\hline Motivation & -0.30 & $-0.78,0.19$ & 0.22 & 0.34 \\
\hline CGI-S & -0.4 & $-1.1,0.2$ & 0.20 & 0.58 \\
\hline Epworth sleepiness scale & -0.9 & $-3.3,1.5$ & 0.46 & 0.22 \\
\hline UPDRS IV (Complications of therapy) & -1.0 & $-2.0,0.0$ & 0.06 & 0.55 \\
\hline UPDRS VI (Schwab and England ADL) & 3.2 & $-1.9, Y 8.3$ & 0.22 & 0.41 \\
\hline
\end{tabular}

a Difference in LS means (pimavanserin-placebo).

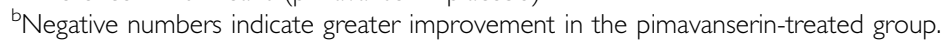

${ }^{\mathrm{C}}$ Test for treatment effect from ANCOVA on the change from baseline score with fixed effects for treatment and center. Baseline score is the covariate. $p$-values are not adjusted for multiple comparisons.

${ }^{d}$ Estimated as the absolute value of the difference in the adjusted mean change from baseline (pimavanserin-placebo) divided by the pooled standard deviation of the mean change from baseline score.

hallucinations (five patients, 16.1\%), dizziness (four patients, $12.9 \%$ ), and fall, headache, confusional state and hypotension each occurring in three $(9.7 \%)$ patients.

Treatment-emergent motor function adverse events occurred infrequently in both treatment arms, were mild, and did not lead to drug discontinuation. They included balance disorder (two pimavanserin-treated patients, 6.9\%), bradykinesia (two placebo-treated patient, 3.2\%), freezing phenomenon (two pimavanserin-treated patients, 6.9\%), on and off phenomenon (one pimavanserin-treated patient, $3.4 \%$ ), tremor (one placebo-treated patient, 3.2\%), and walking disability (one placebo-treated patient, $3.2 \%$ ).

With regard to serious adverse events, there were four events reported in four patients who were not considered to be related to study drug (volvulus (placebo), organic delusional psychosis with paranoia (placebo), fractured ankle (pimavanserin), and junctional rhythm (pimavanserin)). There were no trends or clinically meaningful changes in clinical laboratories, vital signs, 12-lead ECG measurements, or neurological measurements.

\section{DISCUSSION}

The major findings of this placebo-controlled study are that pimavanserin, a selective $5-\mathrm{HT}_{2 \mathrm{~A}}$ inverse agonist, was tolerable and efficacious, by some, but not all measures, for the treatment of PDP. There were no significant differences in the change from baseline in Parts II and III of the UPDRS score and the adverse event profiles in the two treatment groups. Most importantly, pimavanserin showed advantages in antipsychotic efficacy over placebo in four key measures. Statistically significant improvements were observed in the SAPS global hallucinations and delusions scores, persecutory delusions, and UPDRS-PART I thought disorder item in pimavanserin-treated compared with placebo-treated patients. However, the principal measures of efficacy of antipsychotic response to pimavanserin, the SAPS domain scores, only showed a trend.

\section{Effects on Motor Function and Tolerability}

Clinical trials of drugs to treat PDP customarily make motor symptoms the secondary end point. Tolerability measures were designated as the primary end point in this initial study of pimavanserin in patients with PDP because it is essential to further interest in any putative novel treatment for PDP, given the intolerability of most antipsychotic drugs. This study was adequately powered to show motoric worsening if it had occurred to the extent that has been reported with olanzapine (Goetz et al, 2000). For example, Molho and Factor (1999) have reported marked worsening of motor symptoms with olanzapine in 6 of 12 PDP patients treated with low-dose olanzapine. The duration of this study, 4 weeks, was the same as that in the initial randomized study of the tolerability and efficacy of clozapine in PDP (Parkinson Study Group, 1999).

Motor function and activities of daily living were not adversely affected by pimavanserin, as shown by an absence of a meaningful difference between pimavanserin and placebo on the UPDRS Parts II and III score change from baseline scores. These results are supported by adverse event data and are consistent with a recent report that pimavanserin reduced tremor in an animal model of parkinsonian tremor and reduced levodopa-induced dyskinesias in monkeys rendered parkinsonian with MPTP (1methyl-4-phenyl-1,2,3,6-tetrahydropyridine) (Vanover et al, 2008).

As previously discussed, the antipsychotics commonly used in PDP, clozapine, and quetiapine, have tolerability problems, which limit their acceptability. This was not seen with pimavanserin, whose adverse event profile was found to be clinically similar to placebo. Only occasional mild sedation, no hypotension, and no hypersalivation, the most 
Table 5 Summary of Adverse Events $\geqslant 5 \%$ by System Organ Class and Preferred Term in Study ACP-103-006

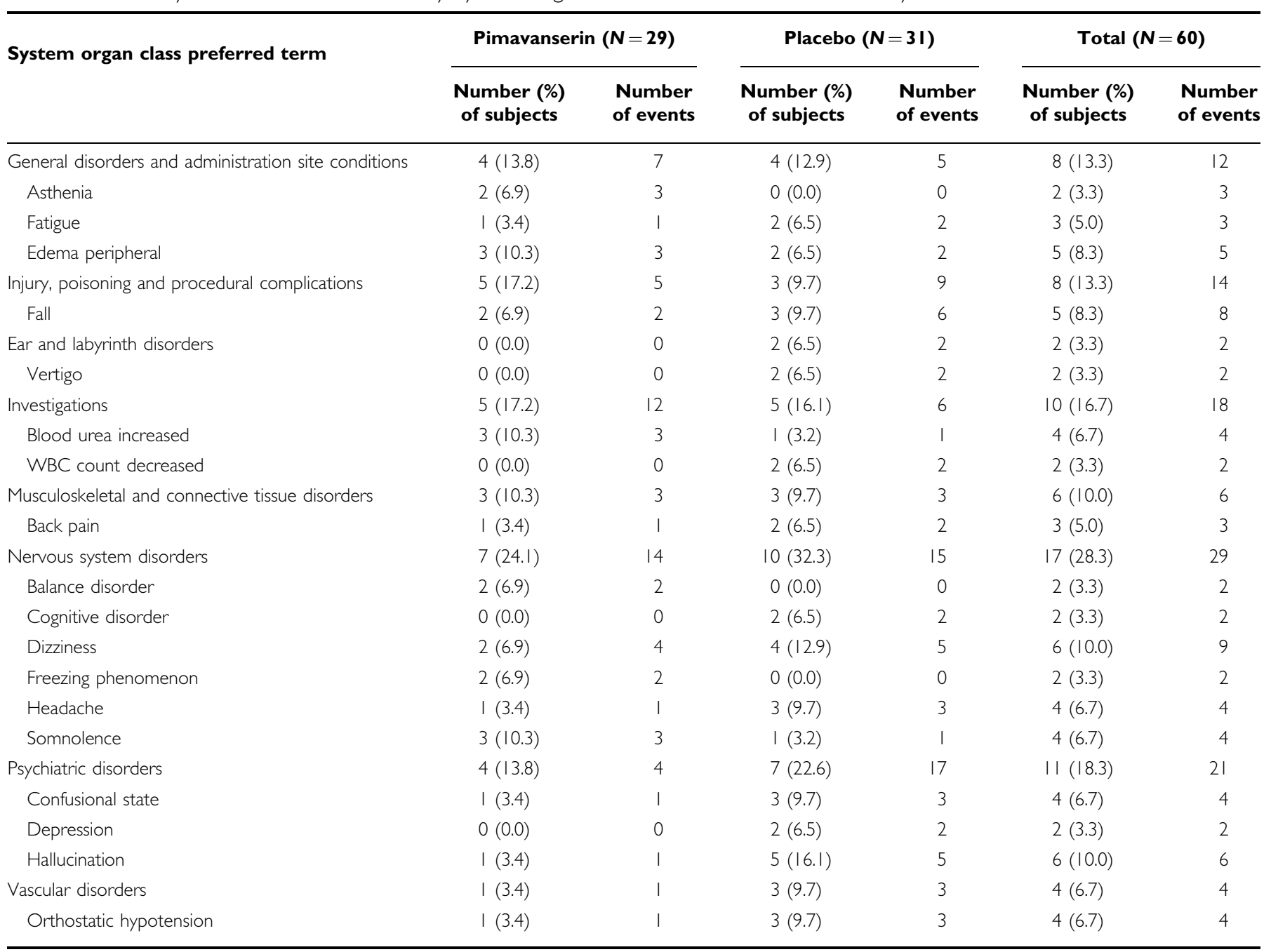

Subjects with more than one event coded to the same system organ class or preferred term were counted only once.

Percentages are based on the number of subjects in the Safety population.

common side effects of clozapine, were noted. Thus, its safety and tolerability profile may confer significant therapeutic advantage over other antipsychotics in the treatment of this fragile patient population.

\section{Effects on Delusions and Hallucinations}

In exploratory analyses of this randomized, placebocontrolled, double-blind trial, pimavanserin showed superiority to placebo in some measures for alleviating delusions and hallucinations in patients with PDP. The SAPS global hallucinations and delusions scores, and the UPDRS Part I item assessing delusions and hallucinations, showed statistically significant advantages for pimavanserin over placebo, despite the small sample size. The global item represents the rater's determination of the overall severity of the symptoms which comprise that domain. The global ratings are the most commonly used SAPS measure for assessing overall severity of psychosis in schizophrenia and other types of psychotic disorders (see Andreasen, 1984; CrespoFacorro et al, 2004; Pressler et al, 2005). In this study, pimavanserin showed a moderate standardized effect size
(0.4-0.6) across these measures of psychosis, suggesting that pimavanserin may confer a meaningful treatment benefit. This is comparable in magnitude with the effect of clozapine over placebo (Parkinson's Disease Study Group, 1999). The SAPS was chosen as the primary measure to assess antipsychotic efficacy for this study because of its extensive use in neuropsychiatric studies for evaluating hallucinations and delusions (Andreasen, 1984). The SAPS is one of four recommended scales by the Movement Disorder Society Task Force on Rating Scales for PD (Fernandez et al, 2008) and has shown sensitivity to assess change in psychosis in previous PDP clinical trials (Parkinson's Study Group, 1999; Goetz et al, 2000).

Examination of the data in Table 3 shows that auditory and visual hallucinations were the most common type of psychotic symptoms in these PDP patients. Although there were improvements in most of the individual hallucination items with pimavanserin, particularly visual and auditory hallucinations, only the global rating for hallucinations reached statistical significance. Paranoid delusions are a leading cause of the need for institutionalization (Wolters, 2006). In this study, delusions of persecution (paranoid 
delusions) were the most prevalent form of delusions, and showed statistically significant improvement in the pimavanserin-treated patients $p=0.009$ ). Similarly, the global delusions rating also showed a statistically significant advantage for pimavanserin $(p=0.02)$.

As in a prior trial of clozapine in PDP (Parkinson's Study Group, 1999), the SAPS domain total, which combines individual item scores with the global rating, was prespecified in the analysis plan. The pimavanserin-placebo difference was greater for delusions than hallucinations, but, in contrast with the global ratings, which also assess severity and disruptiveness, not just frequency, did not reach statistical significance. The difference may have also been due to the variance introduced from assessing specific rare and infrequent subtypes of hallucinations and delusions in the domain analysis, as opposed to the overall clinical judgment provided by the global ratings. Treatment with pimavanserin produced 31.4 and $36.8 \%$ decreases from baseline to day 28 in global hallucinations and delusions, respectively, compared with a $9.4 \%$ decrease, and a $4.8 \%$ increase in the placebo-treated patients, respectively. The slight difference in results between the domain and global scores supports the recommendation of Fernandez et al (2008) that the SAPS global hallucinations and delusions items are the most sensitive measure from the SAPS for clinical trials in PDP, where there has been a history of inability to detect differences in small randomized, blinded trials.

In addition to the SAPS, a number of other measures were used to assess antipsychotic efficacy, including the UPDRS Part I, PPRS, and CGI-S. Improvements in these measures, including statistically significant improvement in UPDRS Part I, and the item which is specified to assess delusions and hallucinations, labeled thought disorder, provide additional support for the SAPS analysis.

\section{Strengths and Weaknesses}

Weaknesses of this study include small sample size and relatively rapid dose escalation. The small sample size had the potential to underestimate efficacy. As it takes between 10 and 14 days to reach steady state with pimavanserin, escalating the dose after 1 week of treatment may have led to some patients having their dose increased before the 20-mg dose had sufficient time to achieve full efficacy. A strength of this study is the exclusion of patients with previous psychiatric disorders and dementia, thereby minimizing the heterogeneity of the study population. This study met all the criteria used by Frieling et al (2007) for inclusion in their meta-analysis of PDP treatment studies: double-blind, randomized, and placebo-controlled. However, we did not assess the time of onset of the delusions or hallucinations in relation to the duration of treatment with L-DOPA. There is evidence that patients with PDP who develop visual hallucinations within the first 3 months of L-DOPA treatment may have an underlying psychiatric disorder, early Lewy body disease, or Alzheimer's disease (Goetz et al, 1998). We did not assess the efficacy of pimavanserin in these patients or those not receiving dopaminomimetic drugs.
Mechanism of Action: 5- $\mathrm{HT}_{2 \mathrm{~A}}$ Receptor Blockade and Diminished Effect of Limbic DA Receptor Stimulation

The ability of pimavanserin to significantly improve hallucinations and delusions in patients with PDP supports the hypothesis that blocking $5-\mathrm{HT}_{2 \mathrm{~A}}$ receptors is an effective means of diminishing positive symptoms in this condition (Meltzer et al, 1995). This hypothesis was based on the supposition that at the low plasma levels of clozapine needed to treat PDP, effective striatal and cortical DA $\mathrm{D}_{2}$ receptor blockade was not possible but adequate blockade of $5-\mathrm{HT}_{2 \mathrm{~A}}$ receptors could be achieved (Meltzer et al, 1989). Subsequent PET studies with clozapine in patients with schizophrenia are consistent with these conclusions (Wiesel et al, 1990; Nordström et al, 1993, 1995; Kessler et al, 2006).

Enhanced stimulation of $\mathrm{DA}_{2}$ receptors in the nucleus accumbens and other limbic nuclei has been proposed as the basis for psychosis in schizophrenia (for review, see Meltzer and Stahl, 1976) and PDP (for review, see Wint et al, 2004; Papapetropoulos and Mash, 2005). Microdialysis studies have shown that DOI, a predominantly $5-\mathrm{HT}_{2 \mathrm{~A}^{-}}$ receptor-acting agonist, can enhance the ability of amphetamine to increase DA efflux in the nucleus accumbens, an effect blocked by ritanserin, which has $5-\mathrm{HT}_{2 \mathrm{~A}}$ and $5-\mathrm{HT}_{2 \mathrm{C}}$ blocking properties (O’Neill et al, 1999; Kuroki et al, 2003). This effect of $5-\mathrm{HT}_{2 \mathrm{~A}}$ receptor stimulation should also be blocked by pimavanserin. Infusion of DOI itself into the nucleus accumbens enhances accumbens DA efflux, an effect blocked by the simultaneous infusion of the selective 5- $\mathrm{HT}_{2 \mathrm{~A}}$ antagonist, M100907 (Yan, 2000; Schmidt et al, 1992). It is also possible that blockade of $5-\mathrm{HT}_{2 \mathrm{~A}}$ receptors located on ventral tegmental DA neurons, which project to limbic and cortical regions (Nocjar et al, 2002), may also contribute to the antipsychotic action of pimavanserin by diminishing the activity of these neurons, which leads to decreased limbic and cortical DA efflux (Pessia et al, 1994; Prisco et al, 1994). Blockade of cortical $5-\mathrm{HT}_{2 \mathrm{~A}}$ receptors might also mediate some of the effects of pimavanserin to attenuate the effects of L-DOPA based on the evidence that cortical $5-\mathrm{HT}_{2 \mathrm{~A}}$ receptor stimulation may increase the activity of ventral tegmental DA neurons through corticotegmental projections (Pehek et al, 2001).

The ability of pimavanserin to attenuate delusions and hallucinations, as reported here, is consistent with other pharmacological (Nichols, 2004) and genetic evidence that delusions and hallucinations may result from increased 5- $\mathrm{HT}_{2 \mathrm{~A}}$ receptor stimulation. (Holmes et al, 1998; Assalt et al, 2004; Lam et al, 2004; Pritchard et al, 2008). It should be noted that there is extensive evidence for the loss of 5-HT neurons in PD (D'Amato et al, 1987), including the loss of 5-HT nerve terminals and diminished levels of 5-HT transporter binding sites in the striatum. Loss of presynaptic 5-HT neurons would be expected to cause supersensitivity of post-synaptic $5-\mathrm{HT}_{2 \mathrm{~A}}$ receptors, whose density has been reported in one study to be reduced by $50 \%$ (Chen et al, 1998), whereas the 5- $\mathrm{HT}_{1 \mathrm{~B}}$ (Castro et al, 1998; Waeber and Palacios, 1989) and 5- $\mathrm{HT}_{4}$ (Reynolds et al, 1995) receptor density has not been found to differ in post-mortem studies of $\mathrm{PD}$ patients from those of comparison groups. However, the number of subjects in these studies is quite small. 
Additional clinical evidence in support of the antipsychotic activity of pimavanserin is provided by a randomized, double-blind, multicenter study of patients with chronic schizophrenia in an acute exacerbation, which found that hallucinations and delusions, as well as total psychopathology, were significantly more rapidly alleviated by the combination of $20 \mathrm{mg} / \mathrm{day}$ of pimavanserin and subeffective doses of risperidone, $2 \mathrm{mg} /$ day, than by a full dose of risperidone, $6 \mathrm{mg} /$ day (NCT00136616; HY Meltzer et al, in preparation). The ability of SR43468B, another selective $5-\mathrm{HT}_{2 \mathrm{~A}}$ antagonist, to decrease positive symptoms in acutely psychotic schizophrenia patients provides further support that selective $5-\mathrm{HT}_{2 \mathrm{~A}}$ antagonists may be effective as antipsychotic agents in schizophrenia (Meltzer et al, 2004).

The findings of this study, if replicated, would suggest that $5-\mathrm{HT}_{2 \mathrm{~A}}$ receptor stimulation may be causally related to psychotic symptoms in PDP and that blockade of 5- $\mathrm{HT}_{2 \mathrm{~A}}$ receptors may be sufficient, in some, but not all cases, to attenuate these symptoms.

\section{CONCLUSIONS}

In conclusion, this small study found that pimavanserin, a selective $5 \mathrm{HT}_{2 \mathrm{~A}}$ inverse agonist, was safe and well tolerated in patients with PDP. In this patient population, pimavanserin was significantly more effective than placebo, in improving both hallucinations and delusions, as measured by the SAPS global hallucinations and delusions items, and the UPDRS thought disorder measure. These results require further confirmation because there was only a trend in the primary measure preselected to assess antipsychotic efficacy. The findings in this study, if confirmed in two ongoing pivotal studies of pimavanserin in PDP (NCT00658567, NCT00477672), may provide an important new treatment option for treating psychosis in patients with $\mathrm{PD}$, as well as adding new evidence for the ability of $5-\mathrm{HT}_{2 \mathrm{~A}}$ receptor blockade to attenuate psychosis in PD.

\section{ACKNOWLEDGEMENTS}

The authors thank the patients who participated in this study; the physicians who cared for them: Susanne Goldstein, James Tetrud, Robert Hauser, Amy Colcher, Jorge Juncos, James Patton, John Murphy, Richard Kishner, Daniel Truong, Rajesh Pahwa, Eric Molho, Ronald Wilson, William Grainger, Sotirios Parashos, and Ramon Gil; and Kimberly A Wilson, Kim Vanover, PhD, Dan van Kammen, MD, PhD, Eve Taylor, PhD, David Weiner, MD and Robert E Davis, $\mathrm{PhD}$, for their individual support of the study.

\section{DISCLOSURE}

Dr Meltzer reports receiving research support from ACADIA, Astra Zeneca, Dainippon Sumitomo, Eli Lilly, Janssen, Pfizer, Memory, Ovation, Minster, Roche, and Litmus Molecular Design; he is also an ACADIA shareholder. Dr Friedman reports receiving funds for lectures, research, or consulting from ACADIA, Astra Zeneca, Novartis, Ingelheim Boehringer, Glaxo Smith Kline, Sepracor, Cephalon, and Teva; he is not a shareholder of any pharmaceutical or biotechnology company. Dr Mills, Dr Revell, Ms Williams, Ms Johnson, and Ms Bahr are ACADIA employees and shareholders.

\section{REFERENCES}

Andreasen NC (1984). Scale for Assessment of Positive Symptoms (SAPS). Department of Psychiatry, University of Iowa: Iowa City, IA.

Arana GW (2000). An overview of side effects caused by typical antipsychotics. J Clin Psychiatry 61(Suppl 8): 5-11.

Assal F, Alarcón M, Solomon EC, Masterman D, Geschwind DH, Cummings JL (2004). Association of the serotonin transporter and receptor gene polymorphisms in neuropsychiatric symptoms in Alzheimer disease. Arch Neurol 61: 1249-1253.

Ballard C, Hanney ML, Theodoulou M, Douglas S, McShane R, Kossakowski K et al (2009). The dementia antipsychotic withdrawal trial (DART-AD): long-term follow-up of a randomised placebo-controlled trial. Lancet Neurol 8: 151-157.

Castro ME, Pascual J, Romón T, Berciano J, Figols J, Pazos A (1998). 5-HT1B receptor binding in degenerative movement disorders. Brain Res 790: 323-328.

Chen CP, Alder JT, Bray L, Kingsbury AE, Francis PT, Foster OJ (1998). Post-synaptic 5-HT1A and 5-HT2A receptors are increased in Parkinson's disease neocortex. Ann N Y Acad Sci 861: 288-289.

Chou KL, Borek LL, Friedman JH (2007). The management of psychosis in movement disorder patients. Expert Opin Pharmacother 8: 935-943.

Crespo-Facorro B, Kim JJ, Chemerinski E, Magnotta V, Andreasen NC, Nopoulos P (2004). Morphometry of the superior temporal plane in schizophrenia: relationship to clinical correlates. J Neuropsychiatry Clin Neurosci 16: 284-294.

Cummings JL, Mega M, Gray K, Rosenberg-Thompson S, Carusi DA, Gornbein J (1994). The Neuropsychiatric Inventory: comprehensive assessment of psychopathology in dementia. Neurology 44: 2308-2314.

D'Amato RJ, Zweig RM, Whitehouse PJ, Wenk GL, Singer HS, Mayeux R, Price DL, Snyder SH (1987). Aminergic systems in Alzheimer's disease and Parkinson's disease. Ann Neurol 22: 229-236.

Diederich NJ, Fénelon G, Stebbins G, Goetz CG (2009). Hallucinations in Parkinson disease. Nat Rev Neurol 5: 331-342.

Factor SA, Feustel PJ, Friedman JH, Comella CL, Goetz CG, Kurlan $\mathrm{R}$ et al (2003). Longitudinal outcome of Parkinson's disease patients with psychosis. Neurology 60: 1756-1761.

Fenelon G, Mahieux F, Huon R, Ziegler M (2000). Hallucinations in Parkinson's disease: prevalence, phenomenology and risk factors. Brain 123(Part 4): 733-745.

Fernandez HH, Friedman JH, Jacques C, Rosenfeld M (1999). Quetiapine for the treatment of drug-induced psychosis in Parkinson's disease. Mov Disord 14: 484-487.

Fernandez HH, Aarsland D, Fenelon G, Friedman JH, Marsh L, Tröster AI et al (2008). Scales to assess psychosis in Parkinson's disease: critique and recommendations. Mov Disord 23: 484-500.

Fernandez HH, Trieschmann ME, Friedman JH (2003). Treatment of psychosis in Parkinson's disease: safety considerations. Drug Safety 26: 643-659.

Friedberg G, Zoldan J, Weizman A, Melamed E (1998). Parkinson Psychosis Rating Scale: a practical instrument for grading psychosis in Parkinson's disease. Clin Neuropharmacol 21: 280-284.

Friedman JH (1991). The management of the levodopa psychoses. Clin Neuropharmacol 14: 283-295.

Frieling H, Hillemacher T, Ziegenbein M, Neundörfer B, Bleich S (2007). Treating dopamimetic psychosis in Parkinson's disease: 
structured review and meta-analysis. Eur Neuropsychopharmacol 17: $165-171$

The French Clozapine Parkinson Study Group (1999). Clozapine in drug-induced psychosis in Parkinson's disease. Lancet 353: 2041-2042.

Goetz CG, Blasucci LM, Leurgans S, Pappert EJ (2000). Olanzapine and clozapine: comparative effects on motor function in hallucinating PD patients. Neurology 55: 789-794.

Goetz CG, Fahn S, Martinez-Martin P, Poewe W, Sampaio C, Stebbins GT et al (2007). Movement Disorder Society-sponsored revision of the Unified Parkinson's Disease Rating Scale (MDSUPDRS): process, format, and clinimetric testing plan. Mov Disord 22: 41-47.

Goetz CG, Leurgans S, Pappert EJ, Raman R, Stemer AB (2001). Prospective longitudinal assessment of hallucinations in Parkinson's disease. Neurology 57: 2078-2082.

Goetz CG, Poewe W, Rascol O, Sampaio C, Stebbins GT, Counsell C et al (2004). Movement Disorder Society Task Force report on the Hoehn and Yahr staging scale: status and recommendations. Mov Disord 19: 1020-1028.

Goetz CG, Vogel C, Tanner CM, Stebbins GT (1998). Early dopaminergic drug-induced hallucinations in parkinsonian patients. Neurology 51: 811-814.

Guy W (1976). Clinical global impressions. ECDEU Assessment Manual for Psychopharmacology, revised (DHEW Publ. No. ADM 76-338). National Institute of Mental Health: Rockville, MD. pp 218-222.

Holmes C, Arranz MJ, Powell JF, Collier DA, Lovestone S (1998). 5-HT2A and 5-HT2C receptor polymorphisms and psychopathology in late onset Alzheimer's disease. Hum Mol Genet 7: 1507-1509.

Johns MW (1991). A new method for measuring daytime sleepiness: the Epworth sleepiness scale. Sleep 4: 540-545.

Kessler RM, Ansari MS, Riccardi P, Li R, Jayathilake K, Dawant B, Meltzer HY (2006). Occupancy of striatal and extrastriatal dopamine D2 receptors by clozapine and quetiapine. Neuropsychopharmacology 31: 1991-2001.

Kuroki T, Meltzer HY, Ichikawa J (1999). Effects of antipsychotic drugs on extracellular dopamine levels in rat medial prefrontal cortex and nucleus accumbens. Pharmacol Exp Ther 288: 774-781.

Lam LC, Tang NL, Ma SL, Zhang W, Chiu HF (2004). 5-HT2A T102C receptor polymorphism and neuropsychiatric symptoms in Alzheimer's disease. Int J Geriatr Psychiatry 19: 523-526.

Lewis JA (1999). Statistical principles for clinical trials (ICH 39): an introductory note on an international guideline. Stat Med 18: 1903-1942.

Mancini F, Tassorelli C, Martignoni E, Moglia A, Nappi G (2004). Long-term evaluation of the effect of quetiapine on hallucinations, delusions and motor function in advanced Parkinson disease. Clin Neuropharmacol 27: 33-37.

Meltzer HY, Arvanitis L, Bauer D, Rein W, Meta-Trial Study Group (2004). Placebo-controlled evaluation of four novel compounds for the treatment of schizophrenia and schizoaffective disorder. Am J Psychiatry 161: 975-984.

Meltzer HY, Kennedy J, Dai J, Parsa M, Riley D (1995). Plasma clozapine levels and the treatment of L-DOPA-induced psychosis in Parkinson's disease. A high potency effect of clozapine. Neuropsychopharmacol 12: 39-45.

Meltzer HY, Li Z, Kaneda Y, Ichikawa J (2003). Serotonin receptors: their key role in drugs to treat schizophrenia. Prog Neuropsychopharmacol Biol Psychiatry 27: 1159-1172.

Meltzer HY, Matsubara S, Lee JC (1989). Classification of typical and atypical antipsychotic drugs on the basis of dopamine D-1, D-2 and serotonin2 $\mathrm{p} K_{\mathrm{i}}$ values. J Pharmacol Exp Ther 251: 238-246.

Meltzer HY, Stahl SM (1976). The dopamine hypothesis of schizophrenia: a review. Schizophr Bull 2: 19-76.

Merims D, Balas M, Peretz C, Shabtai H, Giladi N (2006). Rater-blinded, prospective comparison: quetiapine vs cloza- pine for Parkinson's disease psychosis. Clin Neuropharmacol 29: 331-337.

Miyasaki JM, Shannon K, Voon V, Ravina B, Kleiner-Fisman G, Anderson $\mathrm{K}$ et al (2006). Practice Parameter: evaluation and treatment of depression, psychosis, and dementia in Parkinson disease (an evidence-based review): report of the Quality Standards Subcommittee of the American Academy of Neurology. Neurology 66: 996-1002.

Molho ES, Factor SA (1999). Worsening of motor features of parkinsonism with olanzapine. Mov Disord 14: 1014-1016.

Morgante L, Epifanio A, Spina E, Zappia M, Di Rosa AE, Marconi R et al (2004). Quetiapine and clozapine in parkinsonian patients with dopaminergic psychosis. Clin Neuropharmacol 27: 153-156.

Nichols DE (2004). Hallucinogens. Pharmacol Ther 101: 131-181.

Nocjar C, Roth BL, Pehek EA (2002). Localization of 5-HT(2A) receptors on dopamine cells in subnuclei of the midbrain A10 cell group. Neuroscience 111: 163-176.

Nordström AL, Farde L, Halldin C (1993). High 5-HT2 receptor occupancy in clozapine treated patients demonstrated by PET. Psychopharmacology (Berl) 110: 365-367.

Nordström AL, Farde L, Nyberg S, Karlsson P, Halldin C, Sedvall G (1995). D1, D2, and 5-HT2 receptor occupancy in relation to clozapine serum concentration: a PET study of schizophrenic patients. Am J Psychiatry 152: 1444-1449.

Ondo WG, Tintner R, Voung KD, Lai D, Ringholz G (2005). Double-blind, placebo-controlled, unforced titration parallel trial of quetiapine for dopaminergic-induced hallucinations in Parkinson's disease. Mov Disord 20: 958-963.

O’Neill MF, Heron-Maxwell CL, Shaw G (1999). 5-HT2 receptor antagonism reduces hyperactivity induced by amphetamine, cocaine, and MK-801 but not D1 agonist C-APB. Pharmacol Biochem Behav 63: 237-243.

Papapetropoulos S, Mash DC (2005). Psychotic symptoms in Parkinson's disease. From description to etiology. J Neurol 252: 753-764.

Parkinson Study Group (1999). Low-dose clozapine for the treatment of drug-induced psychosis in Parkinson's disease. $\mathrm{N}$ Engl J Med 340: 757-763.

Pehek EA, McFarlane HG, Maguschak K, Price B, Pluto CP (2001). M100,907, a selective 5-HT(2A) antagonist, attenuates dopamine release in the rat medial prefrontal cortex. Brain Res 888: 51-59.

Pessia M, Jiang ZG, North RA, Johnson SW (1994). Actions of 5-hydroxytryptamine on ventral tegmental area neurons of the rat in vitro. Brain Res 654: 324-330.

Pressler M, Nopoulos P, Ho BC, Andreasen NC (2005). Insular cortex abnormalities in schizophrenia: relationship to symptoms and typical neuroleptic exposure. Biol Psychiatry 57: 394-398.

Prisco S, Pagannone S, Esposito E (1994). Serotonin-dopamine interaction in the rat ventral tegmental area: an electrophysiological study in vivo. J Pharmacol Exp Ther 271: 83-90.

Pritchard AL, Harris J, Pritchard CW, Coates J, Haque S, Holder R et al (2008). Role of 5HT 2A and 5HT 2C polymorphisms in behavioural and psychological symptoms of Alzheimer's disease. Neurobiol Aging 29: 341-347.

Rabey JM, Prokhorov T, Miniovitz A, Dobronevsky E, Klein C (2006). Effect of quetiapine in psychotic Parkinson's disease patients: a double-blind labeled study of 3 months duration. Mov Disord 22: 313-318.

Ravina B, Marder K, Fernandez HH, Friedman JH, McDonald W, Murphy D et al (2007). Diagnostic criteria for psychosis in Parkinson's disease: report of an NINDS, NIMH work group. Mov Disord 22: 1061-1068.

Reddy S, Factor SA, Molho ES, Feustel PJ (2002). The effect of quetiapine on psychosis and motor function in parkinsonian patients with and without dementia. Mov Disord 17: 676-681.

Reynolds GP, Mason SL, Meldrum A, De Keczer S, Parnes H, Eglen RM, Wong EH (1995). 5-Hydroxytryptamine (5-HT)4 receptors 
in post mortem human brain tissue: distribution, pharmacology and effects of neurodegenerative diseases (1995). Br J Pharmacol 114: 993-998.

Romrell J, Fernandez HH, Okun MS (2003). Rationale for current therapies in Parkinson's disease. Exp Opin Pharmacother 4: 1747-1761.

Schmidt CJ, Fadayel GM, Sullivan CK, Taylor VL (1992). 5-HT2 receptors exert a state-dependent regulation of dopaminergic function: studies with MDL 100,907 and the amphetamine analogue, 3,4-methylenedioxymethamphetamine. Eur J Pharmacol 223: 65-74.

Shotbolt P, Samuel M, Fox C, David AS (2009). A randomized controlled trial of quetiapine for psychosis in Parkinson's disease. Neuropsychiatr Dis Treat 5: 327-332.

Vanover KE, Betz AJ, Weber SM, Bibbiani F, Kielaite A, Weiner DM et al (2008). A 5-HT2A receptor inverse agonist, ACP-103, reduces tremor in a rat model and levodopa-induced dyskinesias in a monkey model. Pharmacol Biochem Behav 90: 540-544.

Vanover KE, Weiner DM, Makhay M, Veinbergs I, Gardell LR, Lameh J et al (2006). Pharmacological and behavioral profile of $\mathrm{N}$-(4-fluorophenylmethyl)-N-(1-methylpiperidin-4-yl)-N'-(4-(2methylpropylo xy)phenylmethyl) carbamide (2R,3R)-dihydroxy- butanedioate (2:1) (ACP-103), a novel 5-hydroxytryptamine(2A) receptor inverse agonist. J Pharmacol Exp Ther 317: 910-918.

Waeber C, Palacios JM (1989). Serotonin-1 receptor binding sites in the human basal ganglia are decreased in Huntington's chorea but not in Parkinson's disease: a quantitative in vitro autoradiography study. Neuroscience 32: 337-347.

Wang PS, Schneeweiss S, Avorn J, Fischer MA, Mogun H, Solomon DH et al (2005). Risk of death in elderly users of conventional $v s$ atypical antipsychotic medications. $N$ Engl J Med 353: 2335-2341.

Weintraub D, Stern MB (2005). Psychiatric complications in Parkinson disease. Am J Geriatr Psychiatry 13: 844-851.

Wiesel FA, Farde L, Nordström AL, Sedvall G (1990). Central D1- and D2-receptor occupancy during antipsychotic drug treatment. Prog Neuropsychopharmacol Biol Psychiatry 14: 759-767.

Wint DP, Okun MS, Fernandez HH (2004). Psychosis in Parkinson's disease. J Geriatr Psychiatry Neurol 17: 127-136.

Wolters E (2006). PD-related psychosis: pathophysiology with therapeutical strategies. J Neural Transm 71(Suppl): 31-37.

Yan QS (2000). Activation of 5-HT2A/2C receptors within the nucleus accumbens increases local dopaminergic transmission. Brain Res Bull 51: 75-81. 